\title{
A GLACIAC̄̃̃O JEQUITAÍ: UM GUIA ESTRATIGRÁFICO PARA O PRÉ-CAMBRIANO SUPERIOR NO BRASIL
}

\author{
J. G. PARENTI COUTO * e LAURI BEZ *
}

\begin{abstract}
Based on recent radiometric ages determined for the so called "Bambui Group" and on litogical and stratigraphic descriptions of glaciogenic rocks a new stratigraphic division is suggested for the Upper Precambrian in Brazil. A restrition to the Bambui Group is proposed taking its base as defined by the Paraopeba Formation (Carrancas Member included) made up of carbonated (primarily limestones and subordinate dolomites) and pelitic rocks. The rock sequence, composed primarily of dolomites, that crops in the Vazante/Paracatu region is dropped from the base of the Bambui Group. With this statement becomes clear that the several interpretations correlating the Bambui and Macaúbas Groups have to be revised. The correlation of the stromatolites, described by School (1976) in Couto Magalhaes and located below the glaciogenic rocks with the stromatolites that crop in the Vazante/Paracatu region, is possible taking into account as belonging to a same unit and deposited before the Bambui Group. It is easy now to understand that between this unit, located below the tillites, and the Bambui Group, both deposited in warm climate, as showed by the stromatolites, one or more glaciations occurred. The age of this glacial event is comprised by the time interval of $640 \mathrm{~m} . \mathrm{y}$. (age of Bambui Group base above the tillites in Jequitai region) and 950-1,350 m.y (age of the stromatolites below the tillites in Couto Magalhães region). The name Jequitai Glaciation is suggested for this event. As a consequence of the data presented a new approach for mineral exploration in the Upper Precambrian rocks in Brazil has to be worked out.
\end{abstract}

INTRODUÇÃO O presente artigo tem por finalidade difundir algumas conclusões obtidas a partir dos resultados do "Programa Geocronologia do Bambui", desenvolvido pela Metamig desde 1977. O objetivo então proposto era esclarecer as dúvidas existentes sobre a real idade do Grupo Bambuí e unidades relacionadas, facilitando conseqüentemente a prospecção e a pesquisa mineral no âmbito do Estado de Minas Gerais. Os dados relativos às idades estão sendo divulgados em outro artigo nesta mesma Revista (Couto et al. - no prelo). Embora o enfoque final do presente trabalho (revisão estratigráfica) seja de cunho especulativo, acreditamos que o artigo como um todo contribuirá de alguma forma para melhor compreensão da geologia do Brasil.

\section{A GLACIAÇÃO COMO GUIA ESTRATIGRÁFICO}

A prospeç̧ão e a pesquisa do Pré-Cambriano apresentam um sem-número de dificuldades quando se deseja correlacionar unidades portadoras de mineralizações de diferentes sítios, seja em escala regional, seja continental.

Dechow et al. (1976), em trabalho apresentado ao XXIX Congresso Brasileiro de Geologia realizado em Belo Horizonte, desenvolvem uma temática para pesquisa de metais básicos no Brasil, com base em um modelo africano, contribuindo assim, de forma pioneira, para abrir o debate em torno da correlação estratigráfica em escala continental como instrumento de pesquisa mineral. Nesse artigo foram formuladas algumas dúvidas relacionadas aos grupos Bambuí e Macaúbas, cujos tópicos mais significativos são transcritos a seguir:
"Um importante método de correlação entre rochas de idade Pré-Cambriana Superior na África tem sido através de sedimentos de origem glacial. Em ZâmbiaZaire, na África do sudoeste e em Angola, parece que existiram dois períodos de glaciação (Kroner e Rankam ma, 1972) em torno de 720 a 600 m.a."

"Geralmente, considera-se a idade do Bambuí como sendo Pré-Cambriana Superior (Amaral e Kawashita, 1969) mas Cloud e Dardenne, 1973, baseados nas formas dos estromatólitos em comparação com modelos russos, propuseram uma idade de 950-1 300 m.a. e tal informação serviu como dado principal responsável pela hipótese de Pflug (1975) na qual ambos os grupos Macaúbas e Bambuí são de idade Minas e foram deposin tados durante os estágios finais da orogenia Minas (1 000-1 400 m.a.).

"Achamos, com efeito, que a estreita correlação da estratigrafia Katanga Superior na África, acima dos tilitos, com o Bambuí é muito mais que uma simples coincidência para ser ignorada. Ou as glaciaçðes africanas são muito mais velhas ou as glaciaçðes brasileiras são, de fato, do Pré-Cambriano Superior".

BREVES COMENTÁRIOS SOBRE. A EVOLUÇÃO DA ESTRATIGRAFIA DOS GRUPOS BAMBUÍ E MACAÚBAS A denominação "Grupo Bambui" é aplicada a um conjunto de rochas predominantemente pelito-carbonatadas que cobrem ampla porção da região norte-nordeste do Estado de Minas Gerais e adentram-se para os Estados de Goiás e Bahia. Seus limites nesses três Estados têm sido objeto de con- 
trovérsias fundamentadas no não menos controvertido aspecto estratigráfico.

O Grupo Bambuí, originalmente descrito por Rimann em 1917 no extremo sul da ocorrência no Estado de Minas Gerais, já havia sido objeto de estudos por parte de Eschwege, Derby e outros no século passado e início do presente (uma análise ampla sobre o histórico dessa unidade pode ser encontrada no trabalho de Cos. ta et al., 1970). Com Branco e Costa (1961) é que a definição e a divisão do Grupo Bambuí adquirem contornos mais precisos, influenciando os trabalhos subseqüentes. Braun (1968), ao redefinir o Grupo Bambuí, até então chamado de Série, incluiu em sua base uma nova Formação (Paranoá) composta em sua maior parte de quartzitos, acarretando com isso ampla discussão sobre a estratigrafia e a extensão do Bambuí. Conseqüentemente, surgiram correlaçôes entre as unidades predominantemente quartzíticas que se sotopõem em alguns locais à sequência pelito-carbonatada como foi originalmente definida por Branco e Costa (op, cit.).

O Grupo Macaúbas, cujo nome se origina da Formação Macaúbas, de Moraes, 1930 (in Costa et al., 1976), tanto ou mais que o Grupo Bambuí, tem sido objeto de controvérsias relacionadas não só a seus limites e estratigrafia como também envolvendo problemas de definição. O que caracteriza o Grupo Macaúbas, nas diferentes concepções de diversos autores, é a presença de conglomerados tidos como tilitos ou rochas tilóides, os quais, por si só, se tornaram objeto de ampla discussão desde que Branner, em 1919, sugeriu origem glacial para os conglomerados de Jequitaí, descrito por Derby em 1881 (in Hettich, 1977).

\section{A GLACIAÇÃ்O JEQUITAÍ DEFINIDA COM BASE} EM NOVOS DADOS GEOCRONOLÓGICOS A propriedade em se definir como "Glaciação Jequitan" o fenômeno climático ocorrido no Brasil, no Pre-Cambriano Superior, deve-se em primeiro lugar à primazia da sugestão de Branner; em segundo, por situarem-se na região de Jequitaí as provas mais convincentes dessa glaciação e, finalmente, por possibilitar, mais que em quaisquer outros lugares, condiçðes para se precisar o real posicionamento de tal período com base em novos dados geocronológicos do Grupo Bambuí (Couto et al., op. cit.).

Os dados concretos sobre essa glaciação devem-se a Isotta et al. que, em 1969, apresentaram provas convincentes de uma origem glacial para os conglomerados de Jequitaí, conforme assinala Hettich ( $o p$. cit.), que acrescenta, com ampla documentação, novas provas em favor dessa concepção. Assim, a região que bordeja a Serra do Cabral e se estende até pouco ao norte da localidade de Jequitaí, localizada na parte central de Minas Gerais, situa-se como o local onde mais se acumularam provas de uma origem glacial para os inúmeros conglomerados do Pré-Cambriano Superior, tidos como de origem glacial, que ocorrem espalhados pèlos Estados de Minas Gerais, Bahia, Goiás, Mato Grosso do Sul e Mato Grosso.

A idade do Grupo Bambuí, que sempre se constitui em motivo de controvérsias, recebeu com o citado trabalho de Couto et al. uma nova contribuição mercê da qual foi possível avançar os conceitos ora formulados. As idades obtidas para os eventos de sedimentação foram de 590 m.a. para a Formação Pirapora; 620 m.a. para a Formação Três Marias; e 640 m.a. para a Formação Paraopeba, limitada esta às rochas pelitocarbonatadas, sendo este termo composto predominantemente de calcários, com calcários dolomíticos subordinados. Em Sete Lagoas, tais calcários contêm estromatólitos de idade em torno de 650-950 m.a. (Marchese, 1974). As rochas da região de Vazante/Paracatu, com o termo carbonatado composto predominantemente por dolomitos, com estromatólitos de idade em torno de 950-1 350 m.a. (Cloud et al., 1973), foram excluídas da Formação Paraopeba pois estas, naquela região, apresentaram uma idade mínima (metamorfismo) de 680 m.a.

Diante do exposto, o Grupo Bambuí fica restrito à Formação Paraopeba (basal) como foi redefinida por Braun (1968) e com as restriçðes acima; a Formação Três Marias (intermediária), como foi redefinida por Couto (no prelo); e a Formação Pirapora (Superior), como foi definida por Couto et al. (1978). Os conglomerados descontínuos, que em certos locais constituem a base da Formação Paraopeba, são aqui a ela incorporados com a designação de Membro Carrancas. Esses conglomerados de pequena espessura foram originalmente descritos por Branco e Costa (1961) como sendo a base da Formação Carrancas. Oliveira (1967) confirma sua pequena espessura e reconhece seu caráter descontínuo, denominando-os Membro Carrancas, basal de sua Formação Vila da Chapada. Braun (1968), além de confir: mar as observações de Brance e Costa, e Oliveira, reconhece o caráter particular de que tais conglomerados se formaram à custa do embasamento sobre o qual transgrediu a Formação Paraopeba e denominou-os "Fácies Carrancas" sem contudo incorporá-los àquela Formação, apesar de reconhecer essa particularidade.

Esses conglomerados não devem ser confundidos com o embasamento sobre o qual transgride a Formação Paraopeba, principalmente onde esse embasamento se constitui de conglomerados, como, por exemplo, em Jequitaí.

Definida a base do Grupo Bambuí como sendo constituída da Formação Paraopeba (com o membro basal "Carrancas") composta de rochas pelíticas e carbonatadas (calcários e subordinadamente calcários dolomiti$\cos$ ), e excluída a sequência com dolomitos da região de Vazante/Paracatu, torna evidente que as interpretaçðes de diversos autores de que os grupos Bambuí e Macaúbas são correlacionáveis devem ser revistas.

A correlação entre os dolomitos com estromatólitos identificados por Scholl (1976) em Couto Magalhães, no bordo oriental da Bacia do Bambuí no Estado de Minas Gerais, situados abaixo dos tilitos, com os ocorrentes na faixa Paracatu/Vazante torna-se possivel, desde que se considerem como pertencentes a uma mesma unidade e depositada anteriormente ao Grupo Bambuí. Fica claro, portanto, que entre esta unidade situada abaixo dos tilitos e o Grupo Bambuí, ambos depositados em clima quente, atestado pela presença de estromatólitos, ocorreu uma ou mais glaciações. A idade dessa glaciação situar-se-ia, portanto, entre $640 \mathrm{~m}$.a. (idade da base do Grupo Bambuí que se sobrepóe aos tilitos em Jequitaí) e 
950-1 350 m.a. (idade dos estromatólitos que se sotopõem aos tilitos em Couto Magalhães).

Os dados expostos vêm confirmar as observaçð̃es de Oliveira (1967), que já considerava a seqüência portadora de tilitos como sendo uma unidade separada ("Série Jequitaí") por discordâncias das rochas sobrepostas ("Série Bambuí") e sotopostas ("Série Lavras") baseado em critérios estratigráficos e paleoclimáticos.

A IDADE DAS GLACIAÇÕES NA ÁFRICA E AUSTRÁlIA Nos países acima referidos têm sido freqüentemente descritos dois grupos distintos de rochas glaciais ou relacionadas pertencentes ao Pré-Cambriano Superior.

$\mathrm{Na} A$ Árica, o grupo mais antigo que aflora principalmente nas partes sul, central e norte da África do sudoeste pertence a seqüências sedimentares denominadas Grupo Nosib e Grupo Gariep, sendo que o grupo mais jovem ocupa zonas estratigráficas distintas nos sistemas Damara e Nama, sendo admitida uma idade de 720-700 m.a. para a primeira glaciação e uma idade de $650 \mathrm{~m}$.a. para a segunda (Kröner et al., 1973). Rochas de origem glacial também são encontradas no Zaire e em Zâmbia, onde o Grupo Katanga contém dois horizontes, denominados "Grand Conglomérat" e "Petit Conglomérat", cujos escassos dados geocronológicos indicam 950 m.a. para o primeiro e um intervalo de $710-670$ m.a. para o segundo.

Grande parte do continente australiano sofreu glaciação no intervalo de $750-670 \mathrm{~m}$.a., de acordo com Dunn, Thomson e Rankama, 1971 (in Kröner, op. cit.). Dois períodos glaciais são reconhecidos e denominados separadamente. O mais antigo, que começou a aproximadamente 750 m.a., é denominado "Glaciação Sturtian", na parte central e sul da Austrália, e "G̣laciação Moonlight Valley", nos Kimberley; enquanto o mais novo começou em mais ou menos 670 m.a., sendo denominado "Glaciação Marinoan" no sul e de "Glaciação Egan" no noroeste. O tempo total de duração das duas glaciações é estimado em $100 \mathrm{~m}$.a. As glaciações ocorridas no Pré-Cambriano Superior no Brasil, África e Austrália estão sintetizadas no Quadro 1.

Quadro 1 - Quadro comparativo das glaciações do Pré-Cambriano Superior no Brasil, África e Austrália

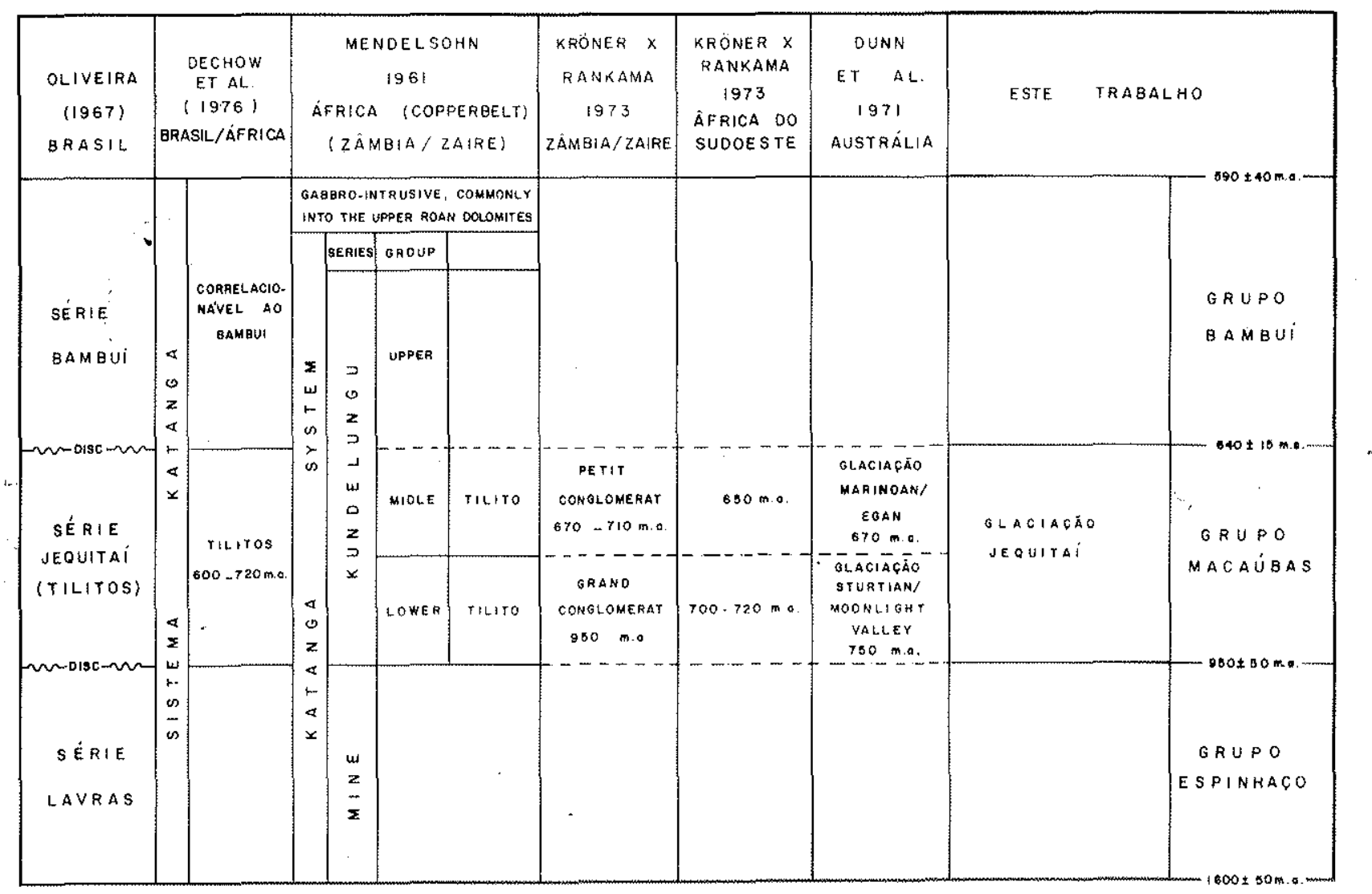


REVISÃO ESTRATIGRÁFICA DO PRÉ-CAMBRIANO SUPERIOR EM MINAS GERAIS COM BASE NA GLACIAÇÃO JEQUITAÍ E NOVOS DADOS GEOCRONOLÓGICOS O Pré-Cambriano Superior (Rifeano) em Minas Gerais está representado por três grupos distintos de rochas, separados por discordâncias e eventos erosivos que marcam os hiatos entre eles. São da base para o topo os grupos Espinhaço, Macaúbas e Bambuí. Em sua maior parte, suas rochas ocupam a bacia hidrográfica do São Francisco a norte do paralelo 20.

O Grupo Espinhaço repousa em discordância sobre um conjunto de rochas vulcânicas, denominadas "Complexo Rio dos Remédios" no Espinhaço setentrional (Costa et al., 1976), cuja idade, obtida por Brito Neves et al. (1979) na regiåo do Espinhaço Meridional, é de 1 770-1 650 m.a.

O Grupo Espinhaço compreende a Série Lavras, de Oliveira (1967); as unidades Média e Superior do Supergrupo Espinhaço, de Costa et al. (1976); A unidade A do Grupo Macaúbas, de Hettich (1977); o Grupo Paranoá, de Alvarenga e Dardenne (1978); e o Grupo Bambuí, de Madalosso e Valle (1978).

A idade da parte superior do Grupo pode ser admitida como 950-1 350 m.a., a partir dos dados obtidos por
Cloud e Dardenne (1973) na Fácies Paracatu/Vazante e por Schöll (1976) na Fácies Couto Magalhães, em dolomitos com estromatólitos. Essa idade pode ser confirmada pelas dataçð̃es levadas a efeito nas rochas básicas intrusivas, as quais acusam uma idade entre 900-1 200 m.a. (Brito Neves et al., 1979).

Embora na Fácies Serra de São Domingos ocorram lentes de dolomitos com estromatólitos, encaixados em quartzitos, não foi ainda dada a publicidade nenhuma datação dessa área.

Sobrepondo-se em discordância às rochas do Grupo Espinhaço, ocorre o Grupo Macaúbas. Este grupo compreende a Série Jequitaí, de Oliveira (1967); o Grupo Macaúbas, basal do Supergrupo São Francisco, de Costa et al. (1976); e as unidades B, C, D, E e F, de Hettich (1977). A idade deste grupo pode ser inferida pelas dataçðes das unidades sobrepostas e sotopostas, podendo numa primeira aproximação ser fixada entre 640-950 m.a. Recobrindo em discordância o Grupo Macaúbas, ocorre o Grupo Bambuí, cuja idade foi fixada entre 590 a $640 \mathrm{~m}$.a. No Quadro 2 estão sintetizadas as alteraçð̃es propostas.

Quadro 2 - Revisão estratigráfica do Pré-Cambriano Superior em Minas Gerais com base na Glaciação Jequitaí e novos dados geocronológicos

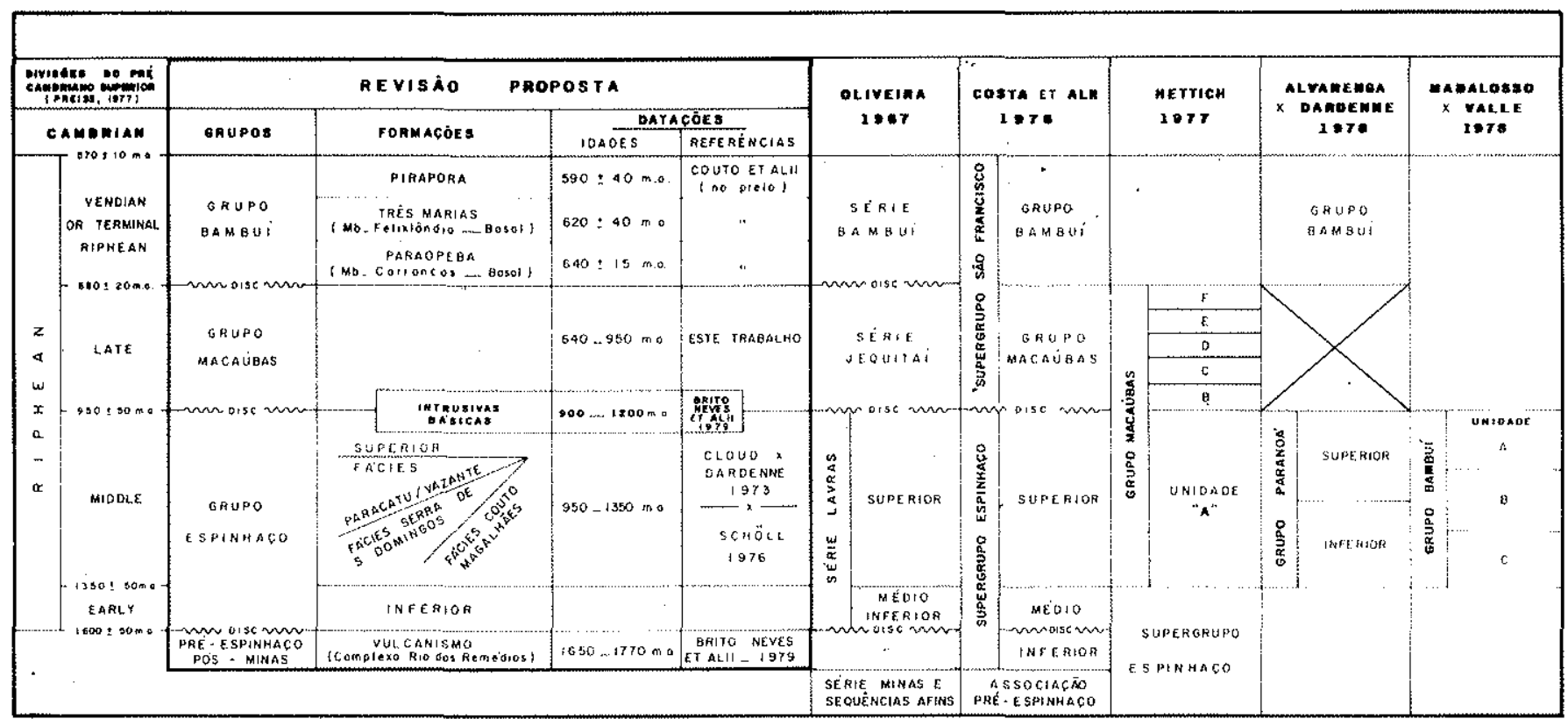




\section{BIBLIOGRAFIA}

ALVARENGA, C. J. S. de e DARDENNE, M.A. - 1978 - Geologia dos Grupos Bambuí e Paranoá, na Serra de São Domingos, MG. Anais do XXX Congresso. Sociedade Brasileira de Geologia, Recife, pp. $546+556$.

BRANCO, J. J. R. c COSTA, M. T, da - 1961 - Roteiro da excursío Belo Horizome/Brasifia. Belo Horizonte, Instituto de Pesquisas Radioativas. (Publicaçào n!: 15). Contributçăo ao Congresso) Brasileiro de Geologia, 14, Belo Horizonte, 1960, p. 117.

BRAUN, OSCAR P. G. - 1968 - Contribuição à estratigrafia do Grupo Bambui. Anais do XXII Congresso. Sociedade Brasileira de Geologia, Belo Horizonte, pp. 155-166.

BRITO NEVES ef al. - 1979 - A evoluçăo geocronológica da Cordisheira do Espinhaco; dados novos e integraçào. Revisła Brasileira de Geociências 9 (1): $71-85$.

CLOUD, P. e DARDENNE, M. A. - 1973 - Proterozoic age of the Bambui Group in Brazil. Geological Society of America Bulletin 84 (5): 1673-1676.

COSTA, L. A M da et al - 1970 - Novos conceitos sobre o Grupo Bambuí sua divisão em tecnogrupos. Boletim de Geologia, Rio de Janeiro (5): 3-34.

COSTA, L. A. M. da et al. - 1976 - Projeto Leste do Tocantins/Oeste do Säo Francisco; relatório final, s.l., Convênio DNPM/CPRM, PROSPEC, v. la.

COUTO, J. G. Parenti - Nota sobre a estratigrafia do Grupo Bambui na região de Felixlândia (Minas Gerais). (No prelo.)

COUTO, J. G. Parenti, ALECRIM J. D. e PINHEIRO, S. O. - 1978 - Reconhecimento geológico-geoquímico das Formaçðes Três Marias e Pirapora (Grupo Bambui). Anais do $X X X$ Congresso. Sociedade Brasileira de Geologia, Recife, v. 5, pp. 2059-2073.

COUTO, J, G. Parenti, CORDANI, U G, KAWASHITA, K, IYER, S. S. MORAES, N. M. P. - Consideraçdes sobre a jdade do Grupo Bambui com base em análises isotópicas de $\mathrm{Sr}$ e $\mathrm{Pb}$ (no prelo.)
DECHOW, E., THOMSON R. C ANDREAZZA, P. - 1976 - Um modelo africano para pesquisa de metais básicos no Brasil. Resumo dos Trabalhos do XXIX Congresso. Sociedade Brasileira de Geologia, Belo Horizonte. (O texto mimeografado contém $17 \mathrm{pp}$., 6 mapas e 1 tabela).

HITTICH, Manfred - 1977 - A glaciação proterozóica no Centro-Norte de Minas Gerais. Revista Brasileira de Geociências 7 (2): 87-101, junho.

KRONER, Alfred e RANKAMA, Kalervo - 1973 - Late precambrian glacio genic sedimentary rocks in southern Africa: a compilation with definitions and correlations. s. L. Separala do Bull. Geol. Soc. Finland 45: 79-102.

MADALOSSO, A. e VALLE, C. R. O. - 1978 - Consideraçðes sobre a estratigrafia e sedimentologia do Grupo Bambuí na regiăo de Paracatu/Morro Agudo (MG). Anais do XXX Congresso. Sociedade Brasileira de Geologia, Recife, pp. 622-634.

MARCHESE, H. C. - 1974 - Estromatolites "gimnosolenidos" en el lado Oriental de Minas Gerais. Revisła Brasileira de Geociências \$: 257-271.

MENDELSOHN, F. - 1961 - w ed. The geology of Nothern Rhodesian Copperbell. London, MacDonald, 1972, sec. imp.

OLIVEIRA, M. A. M. - 1967 - Contribuiça à geologia da parte sul da Bacio do Sáo Francisco edreas adjacentes. Rio de Janeiro, Petrobrás, pp. 71-106 (Coletânea de Relatórios de Exploraçào, 1).

PREISS, W. V. - 1977 - The biostratigrahic potential of precambrian stromatolites. Precambrian Research 5: 207-219.

SCHÖLL, Walter, U, - 1976 - Estromatólitos (conophyton) em dolomitos do Grupo Macaúbas. Resumo dos Trabalhos do XXIX Congresso. Sociedade Brasileira de Geologia, Belo Horizonte.

Recebido em 12 de agosto de 1980 